"This document is the Accepted Manuscript version of a Published Work that appeared in final form in ACS Catalysis, copyright (C) American Chemical Society after peer review and technical editing by the publisher. To access the final edited and published work see [insert ACS Articles on Request author- directed link to Published Work, see http://pubs.acs.org/doi/abs/10.1021/acscatal.5b00132

\title{
Behavior of the Ru-bda water oxidation catalyst covalently anchored on glassy carbon electrodes. Part 2.
}

Roc Matheu, ${ }^{a}$ Laia Francàs, ${ }^{a}$ Petko Chernev, ${ }^{b}$ Mehmed Z. Ertem, ${ }^{c}$ Victor Batista, ${ }^{d, *}$ Michael Haumann, ${ }^{\mathrm{b}}$ Xavier Sala, ${ }^{\mathrm{e}, *}$ and Antoni Llobet, ${ }^{\mathrm{a}, \mathrm{e}, *}$

${ }^{a}$ Institute of Chemical Research of Catalonia (ICIQ), Avinguda Països Catalans 16, 43007 Tarragona, Spain.

${ }^{\mathrm{b}}$ Institut fur Experimentalphysik, Freie Universitat Berlin, Arnimallee 14, D-14195 Berlin, Germany.

${ }^{\mathrm{c}}$ Chemistry Department, Brookhaven National Laboratory, Upton, NY 11973, USA.

${ }^{d}$ Department of Chemistry, Yale University. P.O. Box 208107, New Haven, CT 06520-8107, USA.

e Departament de Química, Universitat Autònoma de Barcelona, Cerdanyola del Vallès, 08193 Barcelona, Spain. 


\begin{abstract}
Reduction of the complex $\left[\mathrm{Ru}\left(\kappa-\mathrm{N}^{2} \mathrm{O}^{2}-\mathrm{bda}\right)(\mathrm{NO})\left(\mathrm{N}-\mathrm{N}_{2}\right)_{2}\right]^{3+}, \mathbf{2}^{3+}, \quad\left(\mathrm{N}-\mathrm{N}_{2}\right.$ is 4-(pyridin-4-yl) benzenediazonium) in acetone allows grafting it onto graphite electrodes (GC). Multiple cycling voltammetric experiments on the same electrode generates a new hybrid material GC-4 with the Ru-aqua complex anchored on the graphite surface. GC-4. While the complex anchored on the graphite surface is an active water oxidation catalyst, as characterized at $\mathrm{pH}=7.0$ via electrochemical techniques and XAS, it has worse performance than in the homogeneous phase and decomposes to form $\mathrm{RuO}_{2}$ at the electrode surface. However, we find that the resulting metal oxide attached to the $\mathrm{GC}$ electrode, $\mathrm{GC}-\mathrm{RuO}_{2}$, is a very fast and rugged heterogeneous water oxidation catalyst with $\mathrm{TOF}_{\mathrm{S}} \mathrm{s}$ of $300 \mathrm{~s}^{-1}$ and TONs $>45000$. The observed performance is comparable to the best electrocatalysts reported, so far, at neutral $\mathrm{pH}$.
\end{abstract}

\title{
Keywords
}

water oxidation catalysis, electrocatalysis, water splitting, Ru complexes, modified graphite electrodes, heterogeneous water oxidation catalysis, $\mathrm{RuO}_{2}$.

TOC

The performance of new hybrid materials based on molecular Ru-bda type of complexes anchored on graphitic surfaces as water oxidation catalysts, is described. The nature of the active species is uncovered based on electrochemical techniques and XAS spectroscopy.

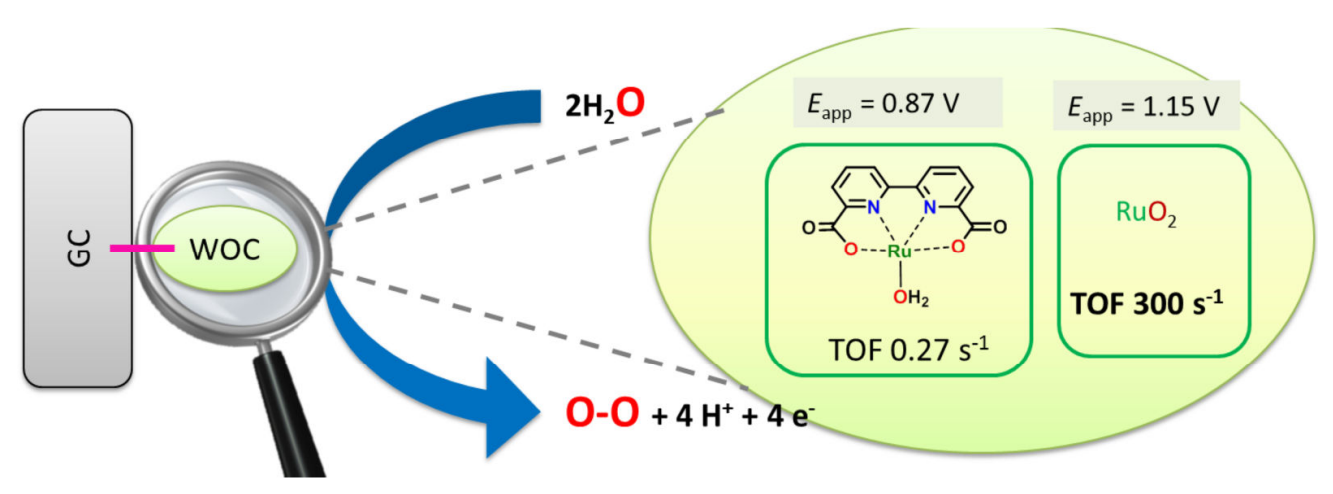




\section{Introduction}

Catalytic water oxidation to molecular dioxygen is one of the key processes in photocatalytic cells that generate solar fuels by solar water-splitting. ${ }^{1}$ In addition, the underlying four-electron/fourproton water oxidation is of biological interest since such reaction takes place at the oxygenevolving $\mathrm{Mn}_{4} \mathrm{Ca}$ complex of photosystem II in green plants and algae. ${ }^{2}$

Significant developments in the field of water oxidation catalysis have emerged over the last few years, including both molecular systems ${ }^{3,4}$ and for metal-oxide catalysts. ${ }^{5,6,7}$ Water oxidation catalysts (WOCs) benefit from molecular toolkit that exploit electronic and steric effects,and can be efficiently combined to generate extremely fast, oxidatively rugged catalysts. ${ }^{8,9,10,11,12,13,14,15,16}$ For such purpose, the effects of ligand perturbations on catalyst performance need to be fully understood, including for example changes in ligand coordination modes, hydrogen-bonding, coordination numbers, inductive effects and site isolation. Finally, molecular WOCs also benefit from an arsenal of spectroscopic techniques that can be applied to molecules and allow to derive detailed information on molecular and electronic structures. ${ }^{17}$ In addition, anchoring WOCs on electrode surfaces is a very attractive strategy for generation of hybrid materials for heterogeneous water oxidation. ${ }^{18,19,20,21,22,23,24}$

Hybrid materials are very attractive since they can provide a large degree of flexibility to build photoelectrochemical cells for water splitting. ${ }^{25,26,27}$ On the other hand, recent contributions have shown that metal oxides obtained from transition metal complexes exhibit highly active water oxidation catalysis. The nature of the transition metal complex as well as the oxide formation protocol strongly influence catalytic performance. . $^{28,6,29,30}$

$\mathrm{RuO}_{2}$ has long been known to be an effective electrocatalytic material for water oxidation to molecular dioxygen. ${ }^{31,32}$ Recent work has focused on the relationship of particle size and shape with catalytic water oxidation performance, at different $\mathrm{pHs}$, including catalysts immobilized on different electrode surfaces. ${ }^{33,34,35,36}$ Here, we complement earlier studies by exploring the catalytic activation of graphite carbon electrodes by using the molecular Ru-aqua complex GC-4 (See Figure 1), obtained by reduction of the corresponding diazonium salt. Furthermore, we analyze the catalytic performance of these new hybrid materials with regard to water oxidation to molecular dioxygen, and the fate of the Ru-complex precursor after catalytic performance. 


\section{Results}

Reduction of the Ru-bda diazonium salt complex $\mathbf{2}^{3+}$ on a glassy carbon electrode in acetone generates the hybrid material GC-4X (where $\mathbf{X}$ refers to a Ru vacant site where a acetone, or an aqua ligand cancoordinate). This material in turn generates the Ru-aqua complex on the surface of the electrode, GC-4, upon several CV cycling experiments in a neat $\mathrm{pH} 7$ phosphate buffer solution, as described in the first part of this paper (Figure 1). ${ }^{37}$ Different graphite electrodes were used to anchor the Ru-bda catalysts, with different shapes and surface area (Figure 2). The amount of mass deposited on the electrode can be controlled by changing the applied potential, the time period for which this potential is applied, or the concentration of the initial diazonium salt $\mathbf{2}^{3+}$. Changing only initial concentration of the diazonium salt, while keeping the same protocol just described, provides an exquisite control of the mass deposited on the electrode surface (See supporting information for further details).

\subsection{The Nature and Activity of the GC-4 Hybrid Materials at Low Potentials.}

The electrochemical properties of GC-4 have been investigated by multiple scanning $\mathrm{CV}$ in water at pH 7.0, as displayed in Figure 3. The upper part of Figure 3 depicts the electrochemical performance of of GC-4 up to $0.90 \mathrm{~V}$ vs. SSCE (all redox potentials reported in this work are versus the SSCE reference electrode unless explicitly mentioned) where the III/II couple at $0.40 \mathrm{~V}$ is clearly seen as well as the electrocatalytic wave associated with the V/IV couple that starts increasing its intensity at approx. $0.75 \mathrm{~V}$. The IV/III couple that is located at $0.60 \mathrm{~V}$ is very weak as in the homogeneous phase. The CV of GC-4 nicely parallels that of $\left[\mathrm{Ru}(\mathrm{bda})(4-\mathrm{Me}-\mathrm{py})_{2}\right]^{10}$ in the homogeneous phase at the same $\mathrm{pH}=7$ and thus corroborates the integrity of the molecular structure even when the complex is anchored on the surface.

Multiple scans, from -0.40 to 0.90 , were carried out to evaluate the electrocatalytic performance of GC-4 and its structural integrity. As can be observed in Figure 3 (top), for increasing number of cycles, the intensity of the catalytic wave decreases as well as the charge under the III/II wave. This observation suggests the presence of a deactivation pathway that slowly reduces the performance of the GC-4 material. Indeed, after 20 cycles, the charge below the III/II couple is reduced by $15 \%$ while the intensity of the electrocatalytic wave decreases by $20 \%$ of its initial value (the second cycle is always taken into consideration for these measurements). These experiments were also performed at 1.00, 1.10 and $1.20 \mathrm{~V}$, as reported in Figure 3 (bottom) and Figures S2 and S3 (SI).

Chronoamperometric measurements were carried out at $E_{a p p}=0.87 \mathrm{~V}$, allowing to calculate an indicative TOF of $0.27 \mathrm{~s}^{-1}$ assuming a 100\% faradaic efficiency (see Figure S4). The approximate TOF $_{i}$ compares well with that of a previously reported Ru-bda complex anchored on GC, following a related immobilization strategy. ${ }^{22}$

The multiple cycling performed at $1.10 \mathrm{~V}$ shows how the intensity of the III/II wave rapidly decreases after 20 cycles to approximately $35 \%$ of its original charge whereas, in sharp contrast 
now, the intensity at $1.10 \mathrm{~V}$ initially decreases but then rapidly increases by $25 \%$. These phenomena are due to the depletion of the $\mathrm{Ru}-\mathrm{OH}_{2}$ active species from the surface of the electrode, concomitant with the generation of new species that are much more active than the $\mathrm{Ru}-\mathrm{OH}_{2}$ but shows a foot of the electrocatalytic wave that is anodically shifted to approximately $1.10 \mathrm{~V}$. These new highly active species are due to the formation of $\mathrm{RuO}_{2}$ on the surface of the $\mathbf{G C}$ electrode, as will be demonstrated in the next section, and will be labeled $\mathbf{G C}-\mathrm{RuO}_{2}$ from now on throughout this manuscript. Interestingly, as is the case for most oxides, ${ }^{5,6,7}$ the $\mathbf{C V}$ of the $\mathbf{G C}^{-\mathbf{R u O}_{2}}$ is featureless except for the electrocatalytic wave.

\subsection{Nature and Activity of the GC-4 Hybrid Materials at High Potentials.}

We have anchored the Ru-aqua complex on large surface glassy carbon thin plates GCp (180 $\mu \mathrm{m} \times$ $15 \mathrm{~mm} \times 25 \mathrm{~mm}$ ) to characterize the nature of the species on the electrode surface during catalytic turnover, following the evolution of reactive species by both electrochemistry and XAS. A similar protocol, as in the case of the GC electrodes, was employed here to generate the corresponding GCp hybrid materials.

Figure 4 shows the electrochemical activity of a GCp-4X material when exposed to 50 consecutive scans, from 0.00 to $1.20 \mathrm{~V}$. The first scan mainly transforms GCp-4X into GCp-4. The increase of the anodic limit to $1.20 \mathrm{~V}$ increases the speed of the transformation of both $\mathbf{G C p}-\mathbf{4 X}$ into GCp-4 and GCp-4 into $\mathbf{G C p}-\mathbf{R u O}_{2}$. This observation is consistent with the featureless response of $\mathbf{G C p}-\mathbf{R u O}_{2}$ except for the large electrocatalytic wave. Thus, the materials generated by electrooxidation involve a mixture of $\mathbf{G C p}-\mathbf{4}$ and $\mathbf{G C p}-\mathbf{R u O}_{2}$ with a relative composition that depends on the number of cycles. Furthermore, the absence of any other wave in the CV reveals the lack of intermediate species in this conversion, indicating a very fast and progressive transformation from $\mathbf{4}$ to $\mathrm{RuO}_{2}$ at the electrode surface. Figure 4 (top left) shows that the intensity under the III/II wave has decreased by about $50 \%$ after 25 cycles, suggesting that about half of the initial amount of $\mathbf{4}$ has been transformed into $\mathrm{RuO}_{2}$.

Modified glassy carbon plates obtained at different voltammetric cycles, labeled $\mathbf{G C p}-\mathbf{4}_{\mathbf{n}}(\mathrm{n}=0,5$, $10,25)$ where " $n$ " indicates the number of cycles (Figure 4 ), were analyzed by XAS. The XAS results obtained for these four samples are summarized in Figure 5 and in the SI. After grafting of [4-K$\mathrm{NO}^{2}\left(\mathrm{H}_{2} \mathrm{O}\right)_{4}$ ] onto GCp electrodes, both the K-edge and EXAFS spectra of GCp- $\mathbf{4}_{0}$ indicated that the overall structure around the Ru center was preserved, although a slight increase of the Ru-N/O bond lengths was observed in GCp-4 $\mathbf{4}_{0}$ (Table S1). Further EXAFS spectroscopic analysis was carried out by combining the spectral data of $\mathbf{G C p}-\mathbf{4}_{5}, \mathbf{G C p}-\mathbf{4}_{10}$ and $\mathbf{G C p}-\mathbf{4}_{25}$, labeled as $\mathbf{G C p}-\mathbf{4}_{\mathrm{av}}$, obtaining the following distances (in $\AA$ ) and coordination numbers $(N)$, in parenthesis: 2.03 (3), 2.51 (1), 2.16 (2) and $3.57(0.8)$. These data again is very similar to the data obtained for $\left[4-\kappa-\mathrm{NO}^{2}\left(\mathrm{H}_{2} \mathrm{O}\right)_{4}\right]^{+}$except for the $3.57 \AA$ distance that manifests the presence of $\mathrm{RuO}_{2}$. 
For increasing numbers of CV scans, an increase of the K-edge energy was observed (Figure 5B) and for $\mathbf{G C p}-\mathbf{4}_{25}$ the increase was about $0.5 \mathrm{eV}$ larger than for $\mathbf{G C p}-\mathbf{4}_{\mathbf{0}}$. Assuming a K-edge shift of $\sim 1.7 \mathrm{eV}$ per Ru oxidation step, ${ }^{37}$ our results suggests that $\sim 30 \%$ of the initial Ru(III) was oxidized to $\mathrm{Ru}(\mathrm{IV}) \mathrm{O}_{2}$. Further, EXAFS analysis revealed a slight decrease of the shorter Ru-N/O bond lengths in GCp- $\mathbf{4}_{\mathrm{n}}$ for increasing CV scan numbers and an increase of the Fourier-Transform (FT) peaks around $3 \AA$. The $\sim 3 \AA$ FT features and the corresponding EXAFS oscillations in the k-range of about 9-12 $\AA^{-1}$ were similar to the spectral features of a $\mathrm{RuO}_{2}$ sample. Accordingly, EXAFS simulations yielded a new Ru-Ru distance of $\sim 3.57 \AA$ in $\mathbf{G C p}-\mathbf{4}_{\text {av }}$ which is similar to the Ru-Ru distance in $\mathrm{RuO}_{2}$. Such distance becomes more prominent for increasing numbers of $\mathrm{CV}$ scans (Figure 5D). The value of $\mathrm{N}_{\mathrm{Ru}-\mathrm{Ru}}$ of $\sim 1.3$ suggests that $\sim 20 \%$ of the ruthenium in $\mathbf{G C p}-\mathbf{4}_{25}$ is present in the form of $\mathbf{R u O}_{2}$. The value obtained here for the transformation of $\mathbf{4}$ to $\mathrm{RuO}_{2}$ on the $\mathbf{G C p}-\mathbf{4}_{25}$ electrode is substantially lower than the one obtained by CV probably due to the fact that not all the generated $\mathrm{RuO}_{2}$ remains tightly attached to the electrode surface and is partially washed off during the rinsing protocol used for the XAS sample preparation.

\subsection{Quantitative Analysis of Performance of Hybrid Materials for Catalytic Water Oxidation.}

The catalytic performance of $\mathrm{RuO}_{2}$, electrodeposited on graphite electrodes by over-oxidation of the molecular precursor 4 , was evaluated by CV and chronoamperometric methodologies. For such purpose, electrodeposition was performed on standard GC disk electrodes achieving surface concentrations close to a monolayer. $\mathbf{G C}$ rods of 5 and $7 \mathrm{~mm}$ diameter were also used to increase the surface area and to drastically decrease the amount of Ru complex anchored on the surface.

Initially, complex $\mathbf{2}^{3+}$ was anchored on the $\mathbf{G C}$ or $\mathbf{G C r}_{\mathbf{x}}(\mathbf{x}=5$ or 7 ) electrodes, following the protocols as previously described. An adequate concentration of the complex was chosen to control the amount of deposited material. Then, the electrode surface was sonicated and rinsed with acetone and cleaned with a phosphate buffer solution at $\mathrm{pH} 7$. Subsequently, the new material was scanned 3 times from $-0.4 \mathrm{~V}$ to $0.6 \mathrm{~V}$ in an aqueous solution at $\mathrm{pH}$ 7. The amount of the complex on the electrode surface was quantified by integrating the charge below the oxidative waves at $0.52 \mathrm{~V}$ and $0.15 \mathrm{~V}$. Finally, a potential of $1.20 \mathrm{~V}$ was applied for 6 minutes to ensure complete conversion from $\mathbf{G C r}_{7}-\mathbf{4 X}$ to $\mathbf{G C r}_{7}-\mathbf{R u O}_{2}$.

The catalytic activity of the new materials was analyzed by $\mathrm{CV}$ and chronoamperometry at $\mathrm{pH}$ 7.0. Figure 6 (left) shows the $\mathrm{CV}$ of $\mathrm{GCr}_{7}-\mathrm{RuO}_{2}$ with a surface concentration of $25 \mathrm{pmols} / \mathrm{cm}^{2}$, exhibiting a huge electrocatalytic wave starting at $1.10 \mathrm{~V}$ that reaches impressive current densities above 1.5 $\mathrm{mA} / \mathrm{cm}^{2}$. Chronoamperometric experiments at $E_{\text {app }}=1.275 \mathrm{~V}\left(\eta=0.70 \mathrm{~V} v \mathrm{~s}\right.$. the $4 \mathrm{e}^{-}$oxidation of water to dioxygen, for 360 seconds) were used to calculate TOF $\mathrm{F}_{\mathrm{i}}$. A plot of TOF $\mathrm{F}_{\mathrm{i}} v$ s. the $\mathrm{RuO}_{2}$ superficial concentration at the electrode is offered in Figure 6 (right) and a respective Tafel plot in Figure S5. As it can be observed in Figure 6 (right) it is impressive to see the large increase of TOF as the $\Gamma$ decreases. In the particular case of $\mathrm{GCr}_{7}-\mathbf{R u O}_{2}$ with $\Gamma=1.0 \mathrm{pmol}$ the $\mathrm{TOF}_{i}$ reaches a value 
close to 300 cycles per second which is among the highest reported in heterogeneous phase, ${ }^{19,22,23,24}$ reaching values very similar to the best ones obtained so far in homogeneous phase. $^{10}$

In order to able to compare the performance of our electrode material with previous works reported in the literature for $\mathrm{RuO}_{2}$ and other oxides deposited at the surface of electrodes, we analyzed the roughness factor (RF) and the water oxidation catalytic activity following the benchmark proposed by Jaramillo et al. ${ }^{38} \mathrm{~A}$ glassy carbon electrode disk $(r=0.20 \mathrm{~cm})$ was used to analyze the double layer capacitance in the absence of faradaic processes to determine the electrochemically active surface area (ECSA) and RF, see figure $\mathrm{S} 6$. Our experiments yielded an RF $=1-2$ (See sup inf) which indicates a surface coverage of $\mathrm{RuO}_{2}$ close or slightly above to one monolayer which is reasonable coming from a very small loading of the initial diazonium salt, $\mathbf{2}^{3+}$. The estimation of the RF enabled us to obtain the specific current density, $\boldsymbol{j}_{s}$, defined as the geometrical current density divided by RF. This parameter allows thus a fair comparison with other electrocatalytic materials since it takes into account the real surface area of the electrode. With regard to catalyst activity we carried out the test recommended by Jaramillo et al. ${ }^{38}$ that consist on evaluating current densities (both $\boldsymbol{j}$ and $\boldsymbol{j}_{\boldsymbol{s}}$ ) as function of potential using a rotating disk electrode (RDE) at $1600 \mathrm{rpm}$ under 1 atmosphere of $\mathrm{O}_{2}$ under steady state conditions using the same GC electrode, see Figure S7. Here our experiments show that to reach a $j_{s}=1 \mathrm{~mA} / \mathrm{cm}^{2}$ at pH $=7.0$ an overpotential $(\eta)$ of $0.65 \mathrm{~V}$ is needed. Under the same conditions $\mathrm{CoPi}^{39,40}$ needs $\eta=1.2 \mathrm{~V}$ at $\mathrm{pH}=$ 0 and $\eta=0.45 \mathrm{~V}$ at $\mathrm{pH}=14$. Under static conditions at $\mathrm{pH}=7$ CoPi needs $\eta=0.58 \mathrm{~V}$ to reach a $j_{s}=$ $0.2 \mathrm{~mA} / \mathrm{cm}^{2}{ }^{41}$ whereas $\mathbf{G C}^{4} \mathrm{RuO}_{2}$ need only $0.50 \mathrm{~V}$ (see the supporting information section for additional details). The latter manifests that the $\mathbf{G C}-\mathbf{R u O}_{2}$ electrode prepared in this work is among the best electrocatalytic materials reported so far.

Finally bulk electrolysis experiments were also carried out using high surface area reticulated carbon sponge electrodes GCs (20 ppi; volume $=1 \mathrm{~cm}^{3}$ ). Following a similar protocol as for the carbon rod electrodes we generated $\mathbf{G C s}-\mathbf{4}$ and $\mathbf{G C s}-\mathbf{R u O}_{2}$. The latter was used to carry out a bulk electrolysis experiment in a two compartment cell with an $E_{\text {app }}=1.15 \mathrm{~V}(\eta=0.6 \mathrm{~V})$ for two hours containing a Clark electrode to measure the molecular oxygen generated in the gas phase. A plot of current intensity and $\left[\mathrm{O}_{2}\right]$ vs. time is presented in Figure $\mathrm{S} 8$. It is impressive to see again that during the first 30 minutes TONs higher than 25000 are achieved with basically $100 \%$ Faradaic efficiency. After 100 minutes the TON reaches a value of 45000 although now the Faradaic efficiency drops, most likely due the oxidation of the carbon sponge electrode as has been observed before. ${ }^{42}$ 


\section{Discussion}

\subsection{Anchored WOCs and the Nature of Ru-bda on Graphitic Surfaces}

Anchoring molecular WOCs on solid surfaces is an attractive strategy to generate hybrid solid-state materials that can be used to carry out heterogeneous water oxidation catalysis. Depending on the nature of the materials, water oxidation anodes or photoanodes can be built. 18,19,20,21,22,23,43,44 Anchored catalysts are very useful for building photoelectrochemical cells for water splitting since they provide a flexible engineering platform. However, one of the most challenging aspects is the proper characterization of the surface-immobilized species before, during, and after catalysis.

A number of WOCs have already been covalently anchored to metal-oxide surfaces, using carboxylate or phosphonate functionalities. ${ }^{18,19,20}$ In addition, a few of them have been anchored on graphite surfaces. ${ }^{22,23,24}$ The graphitic surfaces provide high conductivity, low-cost materials and are readily available in a myriad of conformations. In addition, invariably, every molecular water oxidation catalyst necessarily needs to cycle through a labile $\mathrm{Ru}-\mathrm{OH}_{2}$, or $\mathrm{Ru}-\mathrm{OH}$ type of intermediate species. The oxide surfaces can potentially compete for this bond and thus generate $\mathrm{Ru}-\mathrm{O}_{\text {surface }}$ bonds that in turn deactivate the molecular catalyst. Such deactivation process does not occur on graphitic surfaces. Therefore, from the functional perspective, GC surfaces might have advantages as solid supports when compared to metal-oxides. As a drawback, they are susceptible to oxidation under high applied potentials, so the graphitic surface can be oxidized and the C-C bond between the surface and the molecular catalyst can be broken. However, under 'reasonable' potentials the oxidation of the surface is negligible. In addition, new carbon-based materials such as the $\mathrm{BDD}^{45}$ or nanolTO-RVC $\mathrm{C}^{46}$ are incredibly stable even at very high potentials.

Our strategy was to use diazonium salts attached to the axial ligands that basically maintain the intrinsic electronic properties of the metal center in the original complex. Upon controlled reduction, they readily attach to the surface of the electrode generating hybrid materials. $\mathrm{CV}$ and especially XAS spectroscopy allows thorough characterization of the nature of the anchored species, allowing for characterization of the fresh catalyst before turnover and for monitoring the fate of the catalyst under normal operating conditions. It is satisfying to see that both CV and XAS measurements show that the WOC 4 in homogeneous phase is identical to that anchored on the graphitic surface GC-4.

\subsection{Catalytic Performance of the Anchored Catalysts}

At low potentials, up to the electrocatalytic wave, the GC-4 behaves in a relatively discrete manner achieving TOF $_{i}$ of $0.27 \mathrm{~s}^{-1}$ at $0.87 \mathrm{~V}$. After a few cycles, the catalyst slowly deactivates. This is in sharp contrast with the spectacular performance and stability of the catalyst in the homogeneous

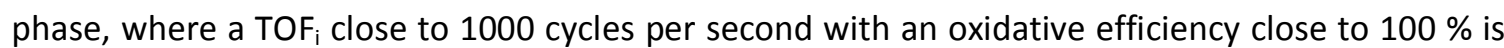
observed under optimized conditions using $\mathrm{Ce}(\mathrm{IV})$ as a primary oxidant. ${ }^{15,16}$ The radically different behavior of the supported catalyst, when compared to the complex in solution, might be due to dimerization of the complex in the homogeneous phase upon reaching the high oxidation state $\mathrm{Ru}(\mathrm{V})$ to generate the RuOORu species via an I2M mechanism and subsequently dioxygen 
evolution. ${ }^{10}$ The low translational mobility of the anchored Ru complex, due to the covalent C-C bond with the graphitic surface, precludes the dimer formation and favors the water nucleophilic attack type of mechanism. Such process has higher activation energy and significant deactivation pathways, as judged by the loss of activity after a few catalytic cycles.

At higher potentials, the electrocatalytic wave shifts anodically by approx. $200 \mathrm{mV}$, indicating that a new material is formed while the original catalyst is depleted. Surprisingly, the newly generated material is extraordinarily active towards water oxidation. Such material is unambiguously characterized as a form of electrodeposited $\mathrm{RuO}_{2}$. The transformation occurs without forming any noticeable reaction intermediates, implying that it is rapidly completed through ligand degradation possibly all the way to $\mathrm{CO}_{2}{ }^{47,}{ }^{48}$ The decomposition might happen in conjunction with ligand loss to the solution. Thus, the anchored molecular catalyst, for instance $\mathbf{G C r}_{7}-4$, acts as a precursor for the generation of $\mathrm{RuO}_{2}$ electrodeposited on the electrode surface, forming $\mathrm{GCr}_{7^{-}}$ $\mathrm{RuO}_{2}$, with TOF $\mathrm{T}_{\mathrm{i}}$ close to $300 \mathrm{~s}^{-1}$ and TONs $>45000$.

At this point, it is of interest to compare the activity of our materials to those that have already been reported in the literature. This is a very difficult task, due the different conditions under which the catalysts are described. To objectively evaluate the performance of the electrocatalytic materials, Jaramillo et al. have described benchmark tests that consists in calculating electroactive surface areas (ECSA), roughness factors (RF) and measurements of current densities ( $\mathbf{j}$ and $\mathbf{j s}$ ) as a function of overpotential values. ${ }^{38}$ Following this benchmark tests, a range of oxides including those of $\mathrm{Co}, \mathrm{Ni}$ and $\mathrm{Ir}$ have been evaluated at $\mathrm{t} \mathrm{pH}=0.0$ and 14.0. These extreme conditions are needed to come up with the best performance for these oxides. Both at $\mathrm{pH}=0$ and $\mathrm{pH}=14$, IrOx turns out to be the best catalyst whereas CoPi performs relatively well at $\mathrm{pH}=14$. Our catalyst exhibits high performance even at $\mathrm{pH}=7.0$, thus we compare our electrocatalytic materials with those of $\mathrm{CoPi}$ at $\mathrm{pH}=7.0$, for which the needed information is available. ${ }^{41}$ The fact that our systems are comparable, or slightly better, in terms of specific current densities than those of CoPi manifests the excellent performance for oxygen evolution of our hybrid electrocatalyst materials. In addition, while $\mathrm{GC}^{-\mathrm{RuO}_{2}}$ works in a neat $\mathrm{pH}=7.0$ electrolyte solution, the CoPi the systems need a $0.5 \mathrm{mM}$ solution of $\mathrm{Co}$ (III) so that a significant amount of $\mathrm{CoOx}$ remains at the electrode.

Another interesting aspect of our system is the inverse correlation of the electrocatalytic activity versus surface concentration. This phenomenon has already been described for metal oxide nanoparticles (NP) and in particular for gold oxides NP, ${ }^{49}$ and has been ascribed to a combination of factors including electronic and geometrical effects. ${ }^{49,50,51}$ From an electronic perspective, the smaller the particle (or nanoparticle) the higher the number of Ru atoms with low coordination sites. An additional influence to the performance can also be due to a synergistic interaction of the electrode surface and the catalyst NP as well as the superficial charge of the NP. From a geometrical perspective, different crystal facets can have different reactivity and the decrease of particle size can also generate an increase of these active facets with regard to the non-active ones. In addition, NP can also have a certain degree of fluxionality that might influence performance. At present, we do not know which one of these factors and at to what extend might 
be responsible for the inverse correlation. Further analysis of this aspect will be reported in the future.

\section{Conclusions}

We have synthesized Ru-bda complexes with axial pyridyl ligands, functionalized with diazonium salts that serve to attach the complexes to graphitic surfaces under reductive treatment. The resulting surface functionalization generates a solid-state material with modest catalytic activity. However, under performance conditions, it readily decomposes to form a highly dispersed $\mathrm{RuO}_{2}$ thin-film exhibiting outstanding electrocatalytic performance for electrocatalytic dioxygen evolution by water-splitting.

\section{ASSOCIATED CONTENT}

Supporting Information. Synthetic procedures and additional experimental, spectroscopic, and electrochemical data. This material is available free of charge via the Internet at http://pubs.acs.org.

\section{AUTHOR INFORMATION}

Corresponding Author

victor.batista@yale.edu; xavier.sala@uab.cat; allobet@iciq.es.

\section{ACKNOWLEDGMENTS}

A.L. thanks MINECO (CTQ-2013-49075-R, SEV-2013-0319) and "La Caixa" foundation for financial support. R.M. thanks "La Caixa" foundation for a PhD grant.

M.H. thanks the Deutsche Forschungsgemeinschaft for financial support (grant Ha3265/6-1) and for a Heisenberg Fellowship and the German Bundesministerium für Bildung und Forschung for funding within the Röntgen-Angström Cluster (grant 05K14KE1). We thank S. Reschke and M. Görlin for help in XAS data collection and M. Nachtegaal at SuperXAS of SLS for excellent technical support

M.Z.E. was funded by a Computational Materials and Chemical Sciences project at Brookhaven National Laboratory under contract DE-AC02-98CH10886 with the U.S. DOE. 
V.S.B. acknowledges supercomputer time from NERSC and financial support as part of the Argonne-Northwestern Solar Energy Research (ANSER) Center, an Energy Frontier Research Center funded by the U.S. Department of Energy, Office of Science, Office of Basic Energy Sciences under Award Number DE-SC0001059.

X.S. thanks MINECO (CTQ2011-26440) for financial support. 


\title{
References
}

\author{
${ }^{1}$ Molecular Water Oxidation Catalysis: A Key Topic for New Sustainable Energy Conversion Schemes. \\ Edited by A. Llobet. 2014 John Wiley and Sons Ltd. ISBN: 9781118413371. \\ 2 Rappaport, F.; Guergova-Kuras, M.; Nixon, P. J.; Diner, B. A.; Lavergne, J. Biochemistry 2002, 41, 8518- \\ 8527.. \\ ${ }^{3}$ Singh, A.; Spiccia, L. Coord. Chem. Rev. 2013, 257, 2607-2622. \\ ${ }^{4}$ Sala, X.; Maji, S.; Bofill, R.; Garcia-Anton, J.; Escriche, L.; Llobet, A. Acc. Chem. Res. 2014, 47, 504-516. \\ 5 Trotochaud, L.; Ranney, J. K.; Williams, K. N.; Boettcher, S. W. J. Am. Chem. Soc. 2012, 134, 17253- \\ 17261. \\ ${ }^{6}$ Smith, R. D. L.; Prévot, M. S.; Fagan, R. D.; Zhang, Z.; Sedach, P. A.; Siu, M. K. J.; Trudel, S.; Berlinguette, C. \\ P. Science 2013, 340, 60-63. \\ ${ }^{7}$ Suntivich, J.; Gasteiger, H. A., Nat. Chem. 2011, 3, 546-550. \\ ${ }^{8}$ Concepcion, J. J.; Tsai, M. K.; Muckerman, J. T.; Meyer, T. J. J. Am. Chem. Soc. 2010, 132, 1545-1557. \\ 9 Yin, Q.; Tan, J. M.; Besson, C.; Geletii, Y. V.; Musaev, D. G.; Kuznetsov, A. E.; Luo, Z.; Hardcastle, K. I.; Hill, \\ C. L. Science 2010, 328, 342-345. \\ 10 Duan, L.; Bozoglian, F.; Mandal, S.; Stewart, B.; Privalov, T.; Llobet, A.; Sun, L. Nat. Chem. 2012, 4, 418- \\ 423. \\ ${ }^{11}$ Karlsson, E. A.; Lee, B.-L.; Åkermark, T.; Johnston, E. V.; Kärkäs, M. D.; Sun, J.; Hansson, O.; Bäckvall, J.- \\ E.; Åkermark, B. Angew. Chem., Int. Ed. 2011, 50, 11715-11718. \\ 12 Zhang, M.-T.; Chen, Z.; Kang, P.; Meyer, T. J. J. Am. Chem. Soc. 2013, 135, 2048-2051. \\ ${ }^{13}$ Lopez, I.; Ertem, M. Z.; Maji, S.; Benet-Buchholz, J.; Keidel, A.; Kuhlmann, U.; Hildebrandt, P.; Cramer, C. \\ J.; Batista, V. S.; Llobet, A. Angew. Chem., Int. Ed. 2014, 53, 205-209. \\ 14 Neudeck, S.; Maji, S.; Lopez, I.; Meyer, S.; Meyer, F.; Llobet, A. J. Am. Chem. Soc. 2014, 136, 24-27. \\ 15 Richmond, C. J.; Matheu, R.; Poater, A.; Falivene, L.; Benet-Buchholz, J.; Sala, X.; Cavallo, L.; Llobet, A. \\ Chem. Eur. J. 2014, 20, 17282-17286. \\ 16 Wang, L.; Duan, L.; Wang, Y.; Ahlquist, M. S. G.; Sun, L. Chem. Commun. 2014, 50, 12947-12950. \\ 17 See for instance: a) Moonshiram, D.; Jurss, J. W.; Concepcion, J. J.; Zakharova, T; Alperovich, I.; Meyer, \\ T. J.; Pushkar, Y. J. Am. Chem. Soc., 2012, 134,4625-4636. . b) Alperovich, I.; Moonshiram, D.; \\ Concepcion, J. J.; Pushkar, Y. J. Phys. Chem. C. 2013, 117, 18994-19001. c) Stull, J. A.; Stich, T. A.; Hurst, J. \\ K.; Britt, R. D. Inorg. Chem. 2013, 52, 4578-4586. \\ ${ }^{18}$ Chen, Z.; Concepcion, J. J.; Jurss, J. W.; Meyer, T. J. J. Am. Chem. Soc. 2009, 131, 15580-15581. \\ ${ }^{19}$ Ashford, D. L.; Lapides, A. M.; Vannucci, A. K.; Hanson, K.; Torelli, D. A.; Harrison, D. P.; Templeton, J. L.; \\ Meyer, T. J. J. Am. Chem. Soc. 2014, 136, 6578-6581. \\ 20 Toma, F. M.; Sartorel, A.; Iurlo, M.; Carraro, M.; Parisse, P.; Maccato, C.; Rapino, S.; Gonzalez, B. R.; \\ Amenitsch, H.; Da Ros, T.; Casalis, L.; Goldoni, A.; Marcaccio, M.; Scorrano, G.; Scoles, G.; Paolucci, F.; \\ Prato, M.; Bonchio, M. Nat. Chem. 2010, 2, 826-831. \\ 21 Tong, L.; Gothelid, M.; Sun, L. Chem. Commun. 2012, 48, 10025-10027. \\ 22 Li, F.; Zhang, B.; Li, X.; Jiang, Y.; Chen, L.; Li, Y.; Sun, L. Angew. Chem., Int. Ed. 2011, 50, 12276-12279. \\ ${ }^{23}$ Mola, J.; Mas-Marza, E.; Sala, X.; Romero, I.; Rodríguez, M.; Viñas, C.; Parella, T.; Llobet, A. Angew. \\ Chem., Int. Ed. 2008, 47, 5830-5832. \\ 24 deKrafft, K. E.; Wang, C.; Xie, Z.; Su, X.; Hinds, B. J.; Lin, W. ACS Appl. Mater. Interfaces 2012, 4, 608 - \\ 613. \\ ${ }^{25}$ Hambourger, M.; Gervaldo, M.; Svedruzic, D.; King, P. W.; Gust, D.; Ghirardi, M.; Moore, A. L.; Moore, T. \\ A. J. Am. Chem. Soc., 2008, 130, 2015-2022. \\ ${ }^{26}$ Youngblood, W. J.; Lee, S.-Y. A.; Kobayashi, Y.; Hernandez-Pagan, E. A.; Hoertz, P. G.; Moore, T. A.; \\ Moore, A. L.; Gust, D.; Mallouk, T. E. J. Am. Chem. Soc., 2009, 131, 926-927. \\ ${ }^{27}$ Li, L.; Duan, L.; Xu, Y.; Gorlov, M.; Hagfeldt, AS.; Sun, L. Chem. Commun., 2010, 46, 7307-7309. \\ ${ }^{28}$ Wang, D.; Ghirlanda, G.; Allen, J. P. J. Am. Chem. Soc. 2014, 136, 10198-10201 \\ ${ }^{29}$ Hong, D.; Jung, J.; Park, J.; Yamada, Y.; Suenobu, T.; Lee, Y.-M.; Nam, W.; Fukuzumi, S. Energy Environ. \\ Sci., 2012, 5, 7606-7616. \\ ${ }^{30}$ Chen, G.; Chen, L.; Ng, S.-M.; Lau, T.-C. ChemSusChem, 2014, 7, 127-134.
}




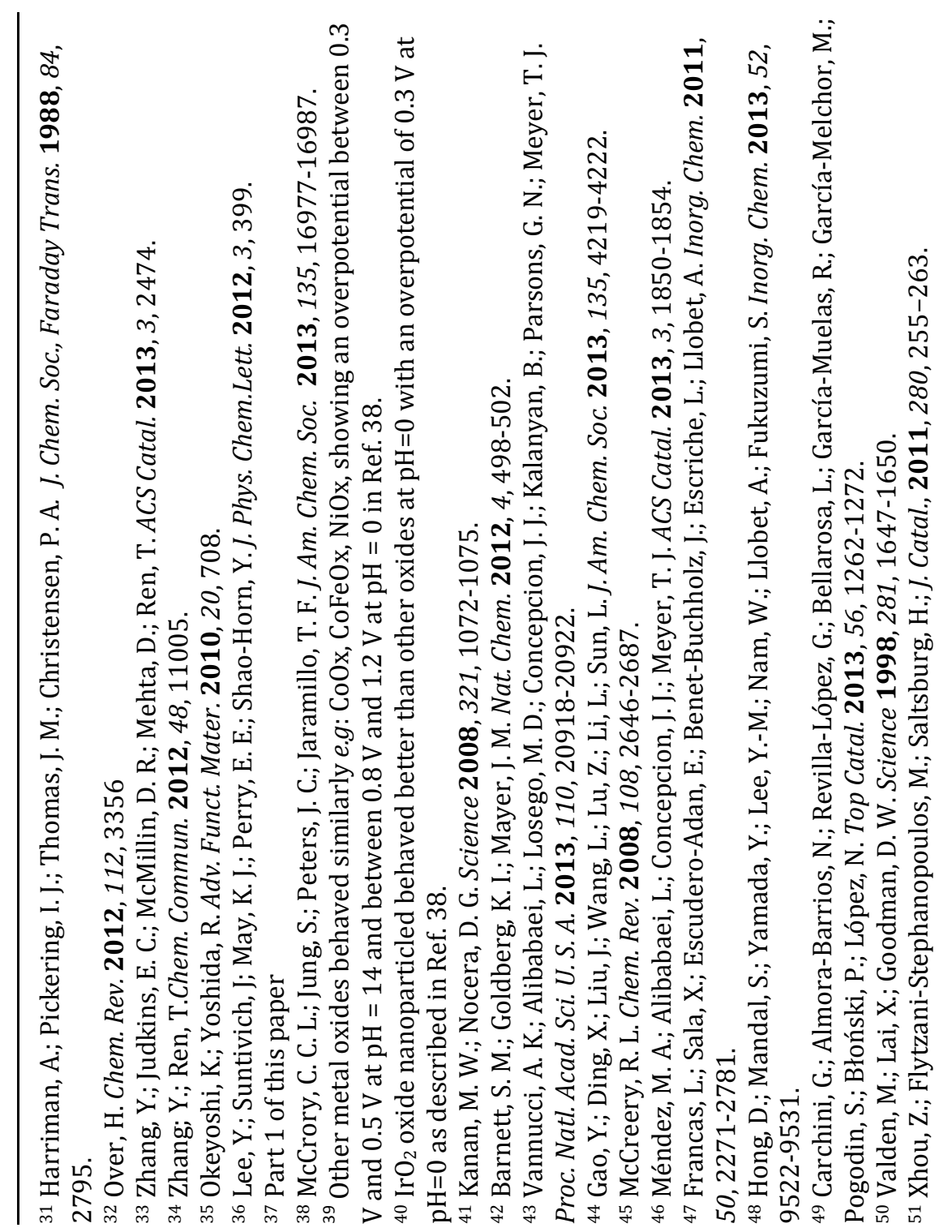




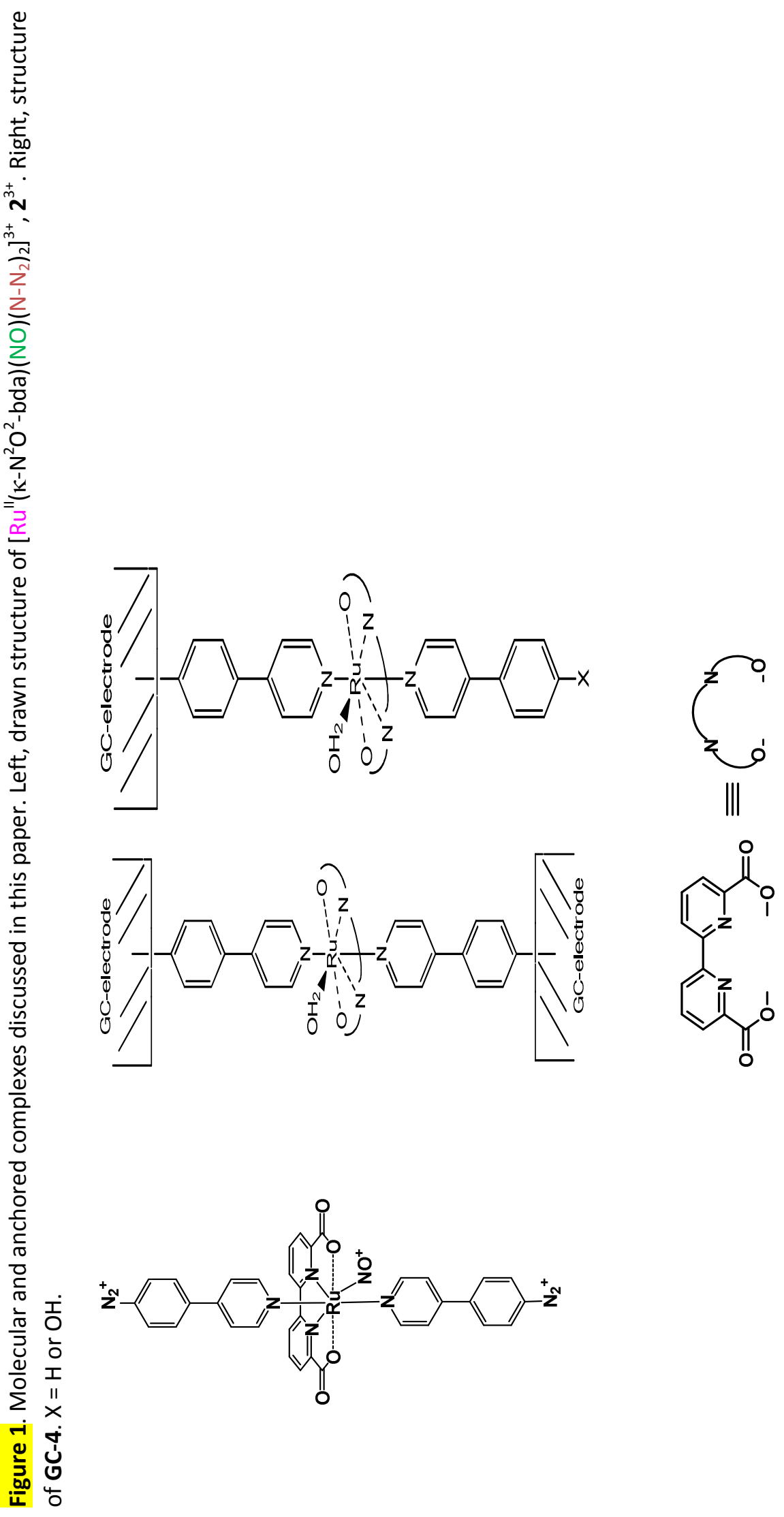




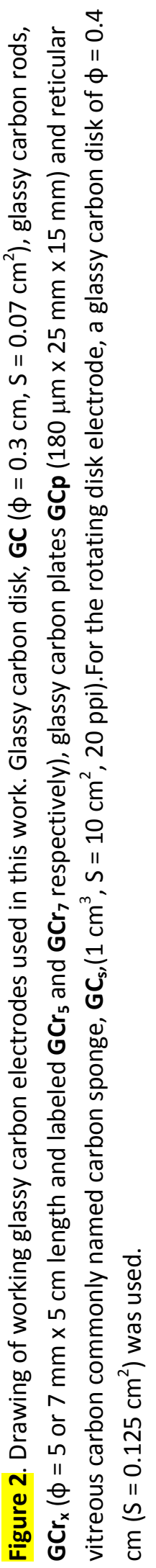

:

$\vdots$

๕

: 

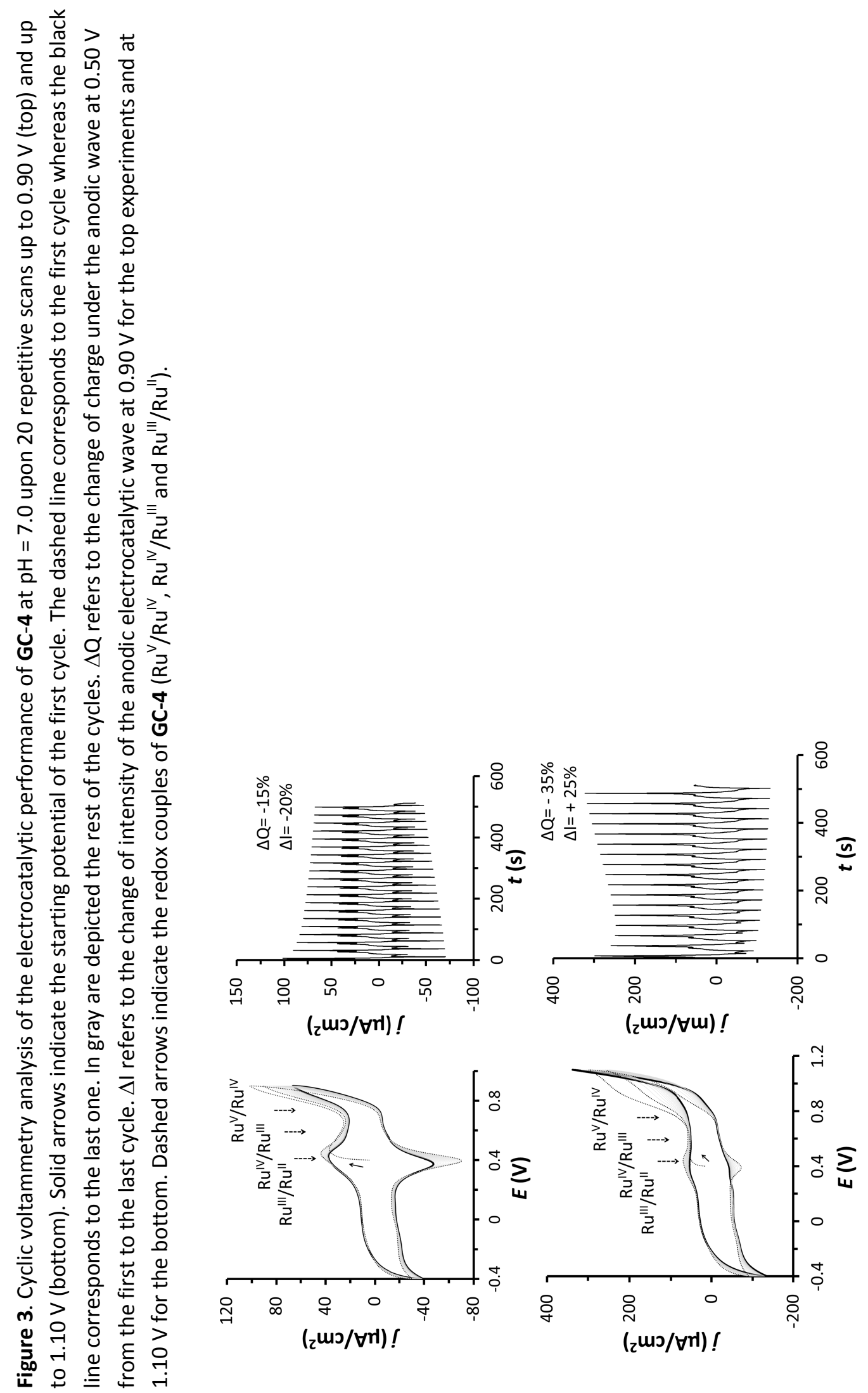


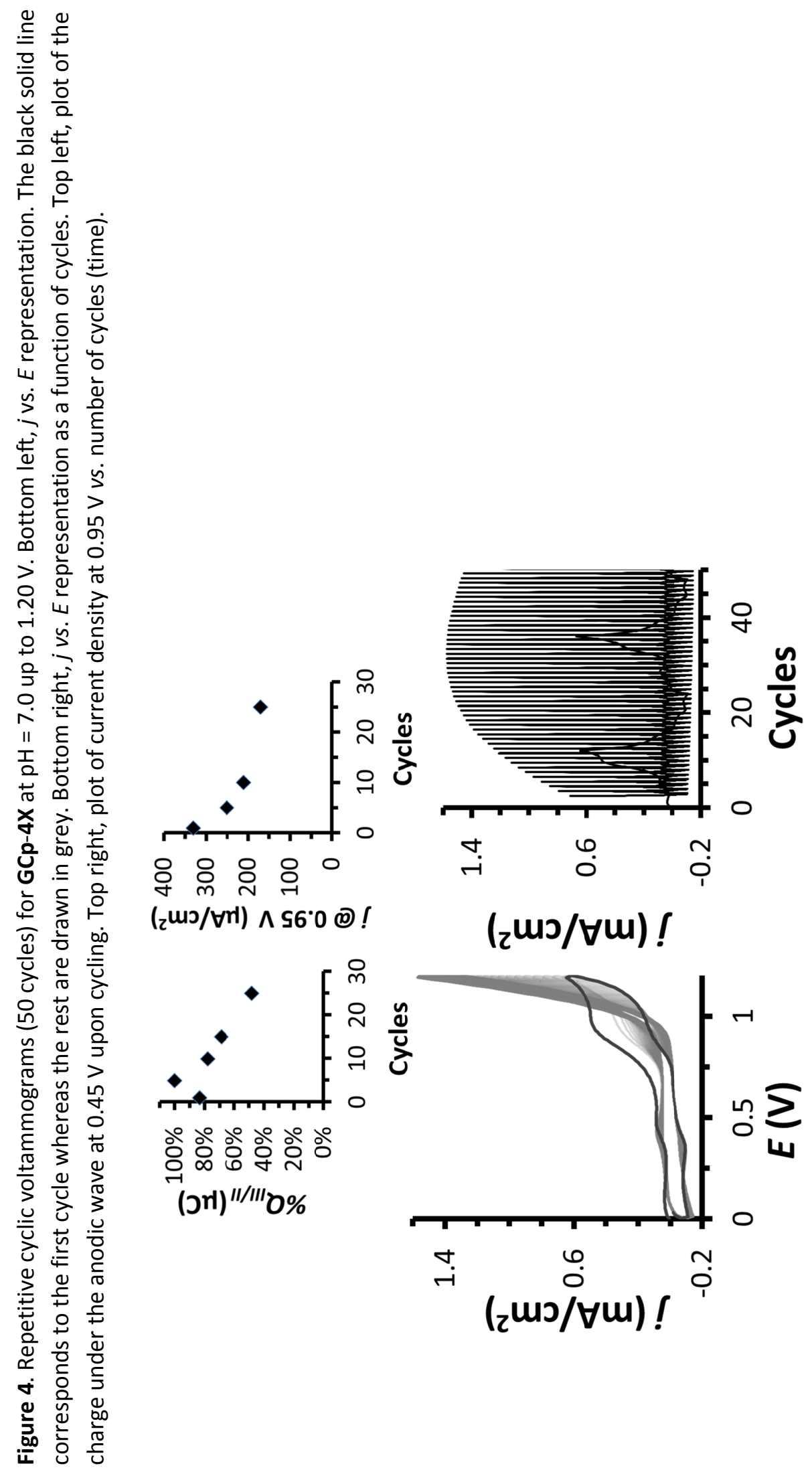




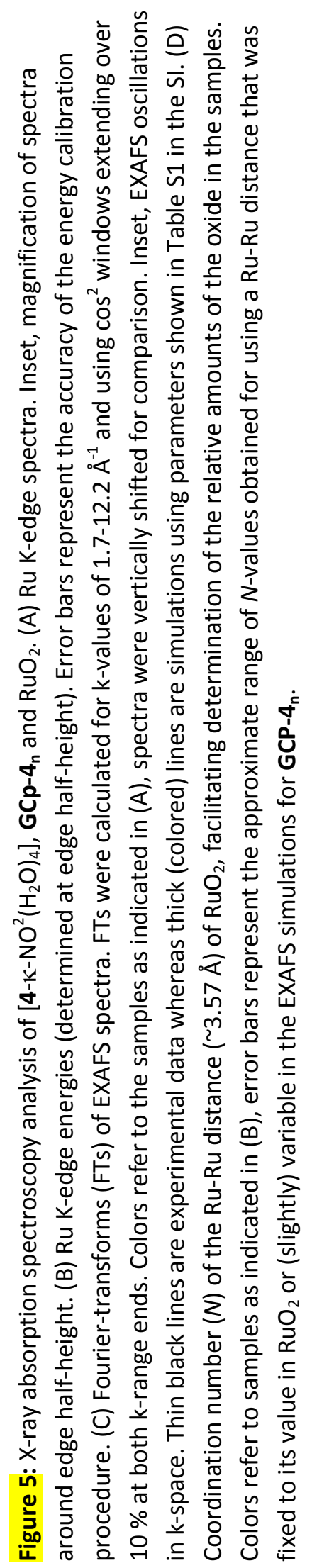



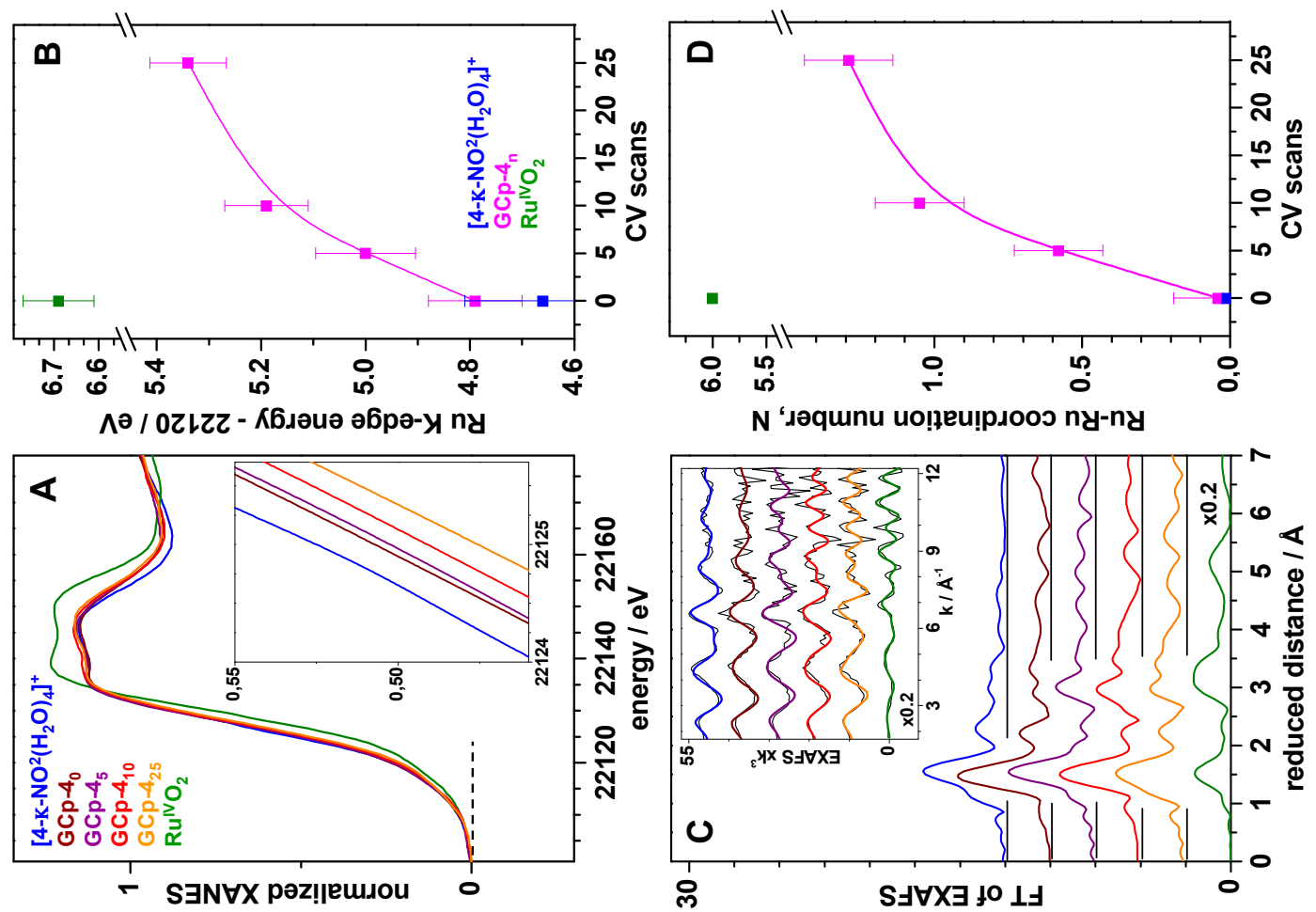


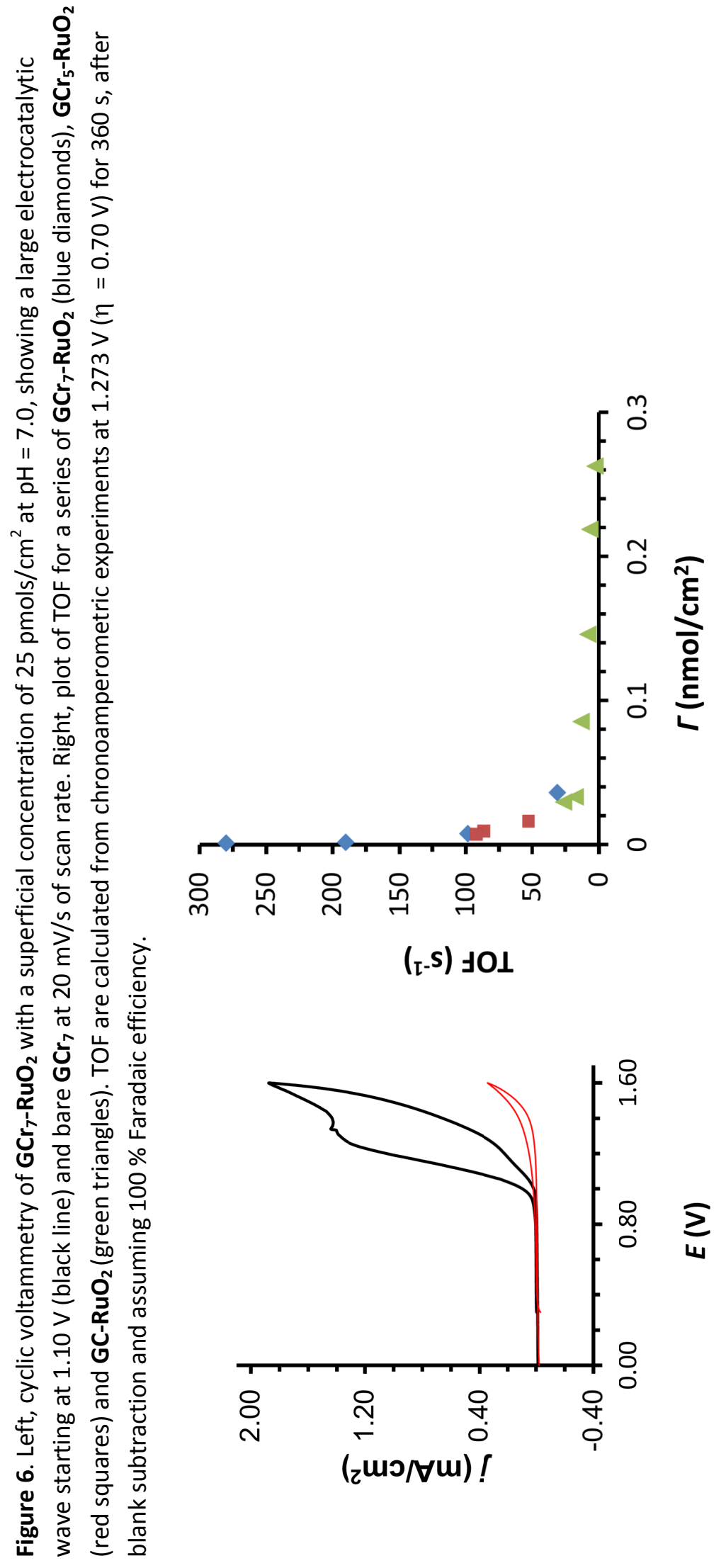

